\title{
On the characterisation of the dynamic compressive behaviour of silicon carbides subjected to isentropic compression experiments
}

\author{
Jean-Luc Zinszner ${ }^{1, a}$, Benjamin Erzar $^{2}$, Pascal Forquin ${ }^{1}$, and François Barthélémy ${ }^{3}$ \\ ${ }^{1}$ Laboratoire Sols Solides Structures - Risques (L3SR), Université Grenoble Alpes, BP. 53, 38041 Grenoble Cedex 9, \\ France \\ ${ }^{2}$ CEA, DAM, GRAMAT, BP. 80200, 46500 Gramat, France \\ ${ }^{3}$ DGA Techniques Terrestres, 18021 Bourges Cedex, France
}

\begin{abstract}
Ceramic materials are commonly used as protective materials particularly due to their very high hardness and compressive strength. However, the microstructure of a ceramic has a great influence on its compressive strength and on its ballistic efficiency. To study the influence of microstructural parameters on the dynamic compressive behaviour of silicon carbides, isentropic compression experiments have been performed on two silicon carbide grades using a high pulsed power generator called GEPI. Contrary to plate impact experiments, the use of the GEPI device and of the lagrangian analysis allows determining the whole loading path. The two SiC grades studied present different Hugoniot elastic limit (HEL) due to their different microstructures. For these materials, the experimental technique allowed evaluating the evolution of the equivalent stress during the dynamic compression. It has been observed that these two grades present a work hardening more or less pronounced after the HEL. The densification of the material seems to have more influence on the HEL than the grain size.
\end{abstract}

\section{Introduction}

Ceramic materials are commonly used as protective materials since the sixties and the Vietnam War [1]. Indeed, thanks to their outstanding hardness and compressive strength [2] combined to their low density, their use in bilayered armour configurations allows, for a same level of protection, significant weight benefit in comparison to monolithic steel plate armours [3]. However, the microstructure of the ceramic may have a strong influence on its penetration resistance [4] and the links between the microstructure and the ballistic efficiency are not fully understood. In order to characterise the dynamic compressive strength of brittle materials, plate impact experiments are generally performed. This kind of experiments allows determining the Hugoniot elastic limit (HEL), i.e. the elastic limit of the material under uniaxial strain state, and the Hugoniot curve which is the loci of all possible equilibrium states under shock loading. Moreover, the determination of yield strength of ceramics with the flyer plate technique requires intrusive techniques like lateral stress gauges embedded in the material. Several tests are necessary to determine accurately shock response of ceramics. In this work, dynamic compression experiments have been performed on two silicon carbide grades using a highpulsed power generator called GEPI. The GEPI machine is a low inductance electric generator, based on the stripline concept and composed of two electrodes separated by dielectric foils [5]. The current, reaching progressively an intensity up to $3.3 \mathrm{MA}$ in nearly $0.5 \mu \mathrm{s}$, generates on the internal faces of the electrodes a high-pressure loading through Laplace effect. Using this generator, the same compressive pulse is applied on the two electrodes.

${ }^{a}$ Corresponding author: jeanluc.zinszner@3sr-grenoble.fr
Thus, by performing a compression experiment on two samples of a same material with different thicknesses, one can use the lagrangian analysis method [6]. This method integrates the conservation equations (mass, momentum, energy) between two velocity signals measured on ceramic samples with different thicknesses and allows accessing to the whole loading path without any assumption. Therefore, a single GEPI experiment with 2 samples is sufficient to determine the dynamic compressive behaviour of ceramics up to more than $20 \mathrm{GPa}$. Two silicon carbide grades have been characterised in order to highlight the links between the microstructure of ceramics and their dynamic compressive behaviour. These grades present a high density, but they have been made using pressureless or spark plasma sintering (SPS). These two processes lead to different grain sizes and hardnesses. The differences of dynamic compressive behaviour observed between the grades provide data on the role of the microstructure at very high loading rates. Moreover, all the experimental data have been compared to numerical results allowing validating the constitutive models. First, a brief description of the microstructural characteristics of the two silicon carbide grades is realised. The principles of the testing machine, the experimental method as well as a description of the lagrangian analysis method are then summarized. In a third part, the experimental results obtained on the two silicon carbides grades are presented before comparing it with numerical results.

\section{Presentation of the two silicon carbide grades}

The two silicon carbide grades are sintered by SaintGobain using pressureless sintering process (PS) or spark plasma sintering process (SPS). They are both sintered in 

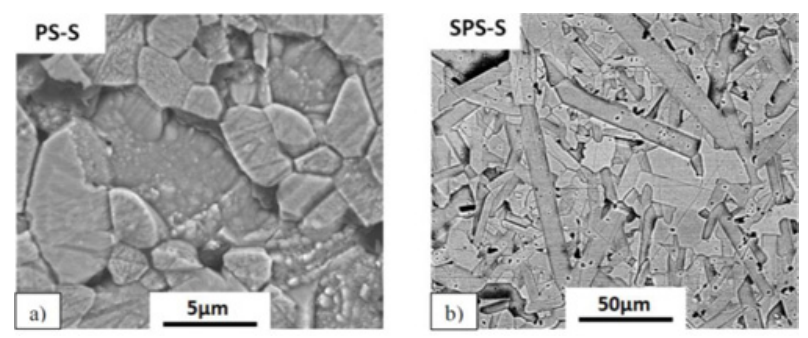

Figure 1. SEM pictures of microstructures from grades (a) PS-S and (b) SPS-S [7].

solid state (SSS) and are called PS-S for the pressureless sintered ceramic and SPS-S for the spark plasma sintered ceramic. These two ceramic grades present different microstructures. The pressureless sintered silicon carbide presents a density greater than $98 \%$ of the theoretical density of the SiC. The microstructure is fine and the grain size is in the range $4-10 \mu \mathrm{m}$ (cf. Fig. 1a). The SPS-S grade presents a density about $99 \%$ the theoretical density. Elongated grains, with size beyond $100 \mu \mathrm{m}$ on the long axis, can be observed in its microstructure (cf. Fig. 1b) [7].

\section{Principle of isentropic compression experiments}

\subsection{Principle of GEPI device}

The GEPI machine is based on High Pulsed Power (HPP) technologies. This generator applies the strip line concept [5]: two plane electrodes made of aluminium, separated by dielectric foils and locally connected by a short circuit (cf. Fig. 2) are subjected to an intense current focused on the load region. In those experiments, the current reaches a maximum intensity of $3.3 \mathrm{MA}$ in nearly $0.5 \mu \mathrm{s}$. The association of current and magnetic field produces Lorentz forces applied on the internal face of the electrodes. The pressure pulse applied to the electrode can be directly computed (cf. Eq. (1)) knowing the evolution of the current with time $I(t)$, the width of the terminal part of the electrode $W$, the vacuum permeability $\mu 0$ and an edge effect factor $\mathrm{kp}$ (near 1).

$$
P_{\text {mag }}(t)=\frac{1}{2} k_{p} \mu_{0}\left[\frac{I(t)}{w}\right]^{2} .
$$

This machine is generally devoted to isentropic compression experiments thanks to the ramp loading. During experiments, the compressive pulse propagates through the electrodes and the ceramic specimens before reaching the $\mathrm{LiF}$ windows. The $\mathrm{LiF}$ window is necessary if one want to perform a dynamic compressive test. When no window is placed after the ceramic, the reflection of the compressive loading on the free surface leads to a tensile fracture of the ceramic. This technique has been used by Erzar and Buzaud [8] to perform shockless spalling tests on alumina.

\subsection{Experimental configuration}

Due to the symmetry of the GEPI configuration (Fig. 2), two specimens can be tested during one GEPI compression

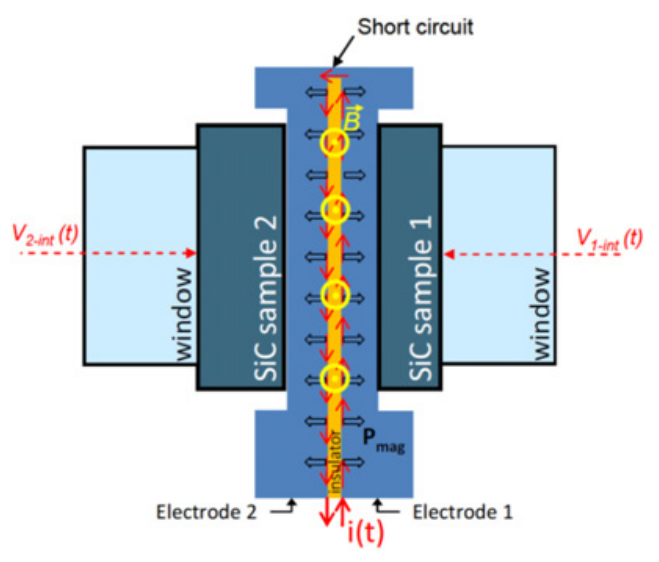

Figure 2. Experimental configuration used to perform GEPI compression tests and allowing using lagrangian analysis method.

test. This configuration also allows using the lagrangian analysis method when a GEPI compression test is performed on two specimens of a same material but having different thicknesses. In this work, one GEPI compression test is performed on each grade. The thicknesses of the specimens are $3 \mathrm{~mm}$ and $4 \mathrm{~mm}$.

The electrode width and the specimen's widths are set to $15 \mathrm{~mm}$. This width allows ensuring a compressive pulse sufficiently high in order to exceed the HEL of the material but also allows ensuring that the interface velocities measured on the two specimens are not affected by radial release waves during the compressive loading.

\subsection{Lagrangian analysis method}

The lagrangian analysis method realised after one GEPI compression test allows determining the whole loading path of the material whereas only one point on this loading path can be obtained after one plate impact experiment. This method is based on the integration of the equations of conservation of mass, momentum and energy. Moreover, this method does not need any assumption on the behaviour of the material. The principle is based on a comparison of velocity signals obtained on two different thicknesses of a material.

For each particular velocity $u_{p}$, an associated wave velocity $C_{L}\left(u_{p}\right)$ can be calculated from times $\Delta \mathrm{t}$ (cf. Fig. 3). This wave velocity then allows us to compute increments of longitudinal stress $\sigma_{x}$, longitudinal strain $\varepsilon_{x}$ (stress and strain being negative in compression) or specific volume associated to each increment of particular velocity following:

$$
\begin{gathered}
d \sigma_{x}=-\rho_{0} C_{L}\left(u_{p}\right) d u_{p} \\
d v=-\frac{1}{\rho_{0} C_{L}\left(u_{p}\right)} d u_{p} \\
d \varepsilon_{x}=\frac{d u_{p}}{C_{L}\left(u_{p}\right)} .
\end{gathered}
$$




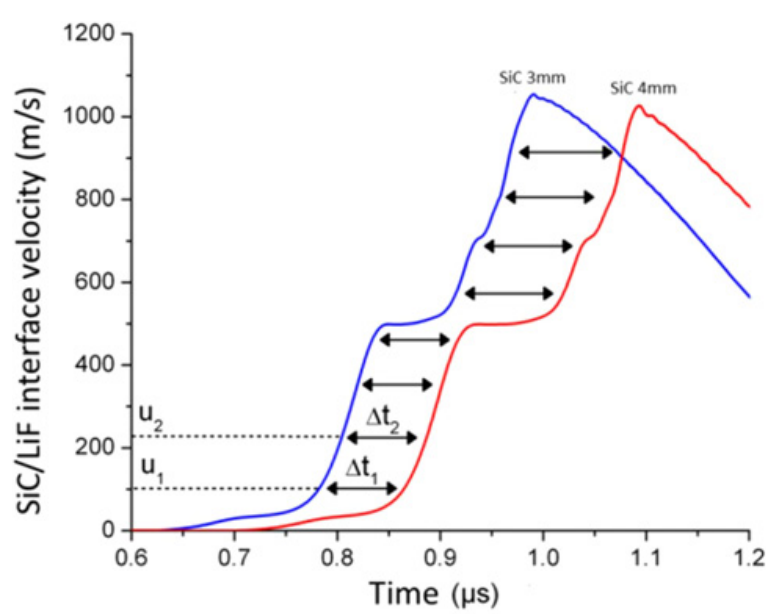

Figure 3. Principle of lagrangian analysis method: determination of the wave velocity $C_{L}\left(u_{p}\right)$ associated to each particular velocity $u_{p}$ (arbitrary origin of time).

It can be noted that a correction based on the mechanical impedances of the ceramic and of the window material have to be realised to determine the particular velocity using the interface velocity measured.

\section{Experimental results}

\subsection{GEPI compression on PS-S grade}

After the GEPI compression test performed on the PS-S grade, one can observe that the velocity signal obtained on the $4 \mathrm{~mm}$ thick specimen presents a shock for velocities comprises between 0 and $460 \mathrm{~m} / \mathrm{s}$ (cf. Fig. 4). Moreover, the rest of this velocity signal presents an uneven profile. It has nevertheless been possible to filter it and to perform a lagrangian analysis using the two velocity signals for velocities comprises between 500 and $850 \mathrm{~m} / \mathrm{s}$. The release signal cannot be used due to the arrival of radial release waves.

In a first time, two methods can be used to determine the HEL of the material. The first one consists in determining the velocity at which a slope change is observed. The HEL is then determined using Eq. (5):

$$
H E L=\rho_{0} C_{L} u_{p}^{L E H}
$$

where $\rho_{0}$ is the initial density, $C_{L}$ is the longitudinal wave velocity in the elastic regime and $u_{p}^{L E H}$ is the particular velocity at the slope change (obtained after correction).

In the case of the PS-S grade, a slope change is observed at about $569 \mathrm{~m} / \mathrm{s}$. That corresponds to a HEL of $14.9 \mathrm{GPa}$.

The second method for determining the HEL consists in exploiting the lagrangian analysis data. By plotting the stress-strain curve, the elastic/inelastic transition is observed starting from 15.1 GPa. These two HEL values are similar but a better confidence is given to results obtained with the lagrangian analysis. In all cases, the uncertainty is evaluated at $\pm 0.2 \mathrm{GPa}$.

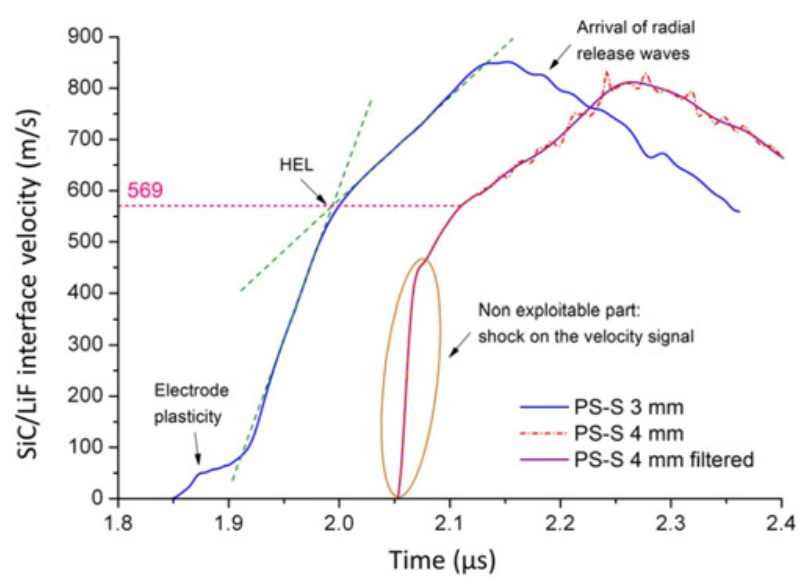

Figure 4. $\mathrm{SiC} / \mathrm{LiF}$ interface velocity signals obtained on the two PS-S specimens after GEPI compression test (arbitrary origin of time).

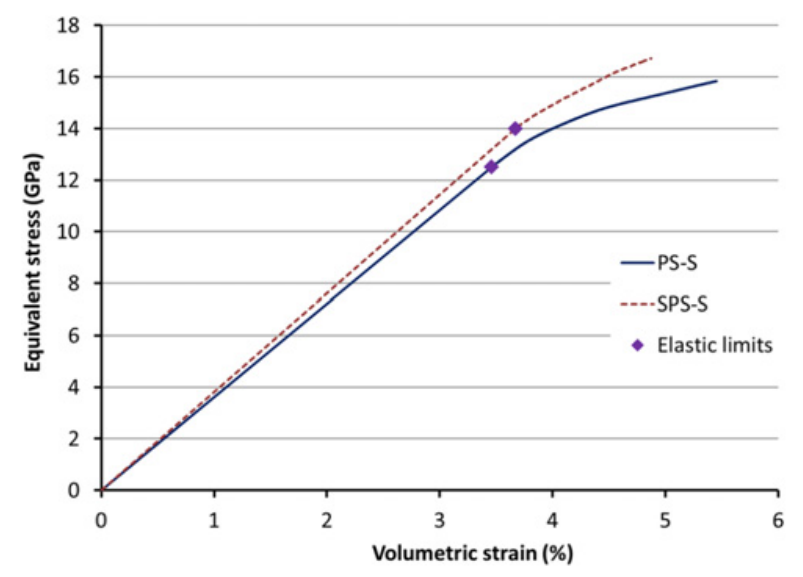

Figure 5. Equivalent stress/strain curves obtained with lagrangian analysis for the two $\mathrm{SiC}$ grades.

The longitudinal stress obtained with the lagrangian analysis method is not sufficient to determine the equivalent stress. However, during a uniaxial deformation or for all triaxial loading such as $\sigma_{y}=\sigma_{z}$, one can write:

$$
\sigma_{e q}=\frac{3}{2}\left(-\sigma_{x}-P\right)
$$

where $\mathrm{P}$ denotes the hydrostatic pressure.

By setting an equation of state for the $\mathrm{SiC}$ material, one can thus evaluate the evolution of the equivalent stress. In the case of the PS-S grade, a low variation of the $K+(4 / 3) \mathrm{G}$ modulus is observed during the elastic compression. Thus, a constant bulk modulus $\mathrm{K}$ is assumed, meaning the pressure is known during the whole experiment. Using Eq. (6), one can deduce the equivalent stress in the ceramic specimen during the compressive loading. The equivalent stress/strain curve obtained is presented in Fig. 5.

One can see that the transition between the elastic and the inelastic behaviour is smooth and that a work hardening of the material is observed after this transition.

A numerical validation has been performed using the Abaqus/Explicit finite element code by imposing as 


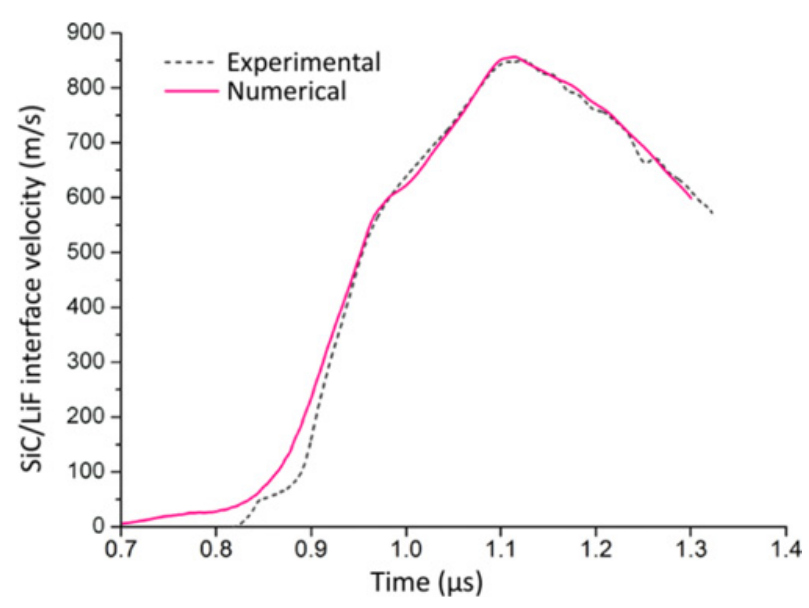

Figure 6. Comparison between experimental and numerical interface velocity signal in the case of the PS-S grade.

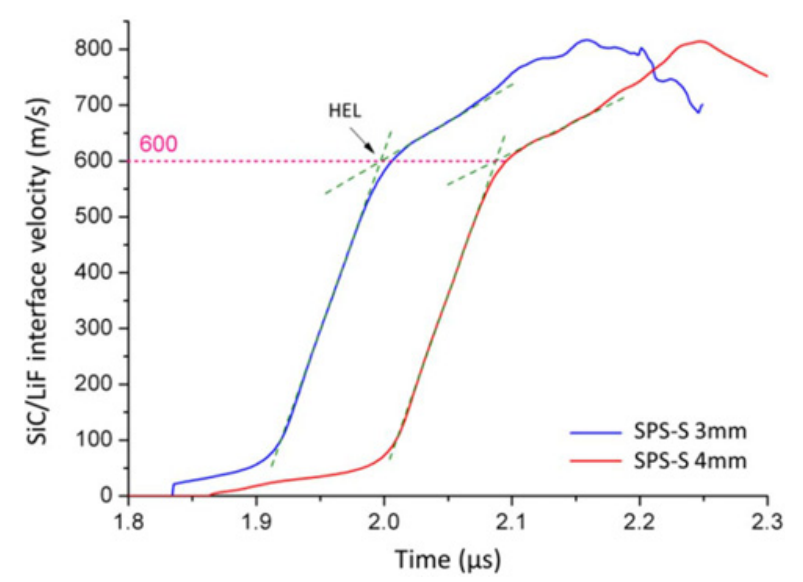

Figure 7. $\mathrm{SiC} / \mathrm{LiF}$ interface velocity signals obtained on the two SPS-S specimens after GEPI compression test (arbitrary origin of time).

plasticity law the stress/strain curve determined above. One can observe that the interface velocity signals obtained experimentally and numerically are similar, validating the plasticity law identified using the lagrangian analysis (cf. Fig. 6).

\subsection{GEPI compression on SPS-S grade}

After the GEPI compression test performed on the SPS-S grade, no shock is observed (cf. Fig. 7). Thus, a lagrangian analysis can be realised using the whole velocity signals. A HEL of $16.8 \mathrm{GPa}$ is obtained with the lagrangian analysis; HEL which is greater than the one of the PS-S grade. This result tends to show that the grain size is not a dominating factor on the HEL of silicon carbides. Indeed, despite the greater grain size of the SPS-S grade and the presence of elongated grains, this grade presents the higher HEL. The better densification of the SPS-S grade is probably the reason why the HEL is higher.

Using Eq. (6), the equivalent stress/strain curve of the SPS-S has been identified. As in the case of the PS-S, a constant bulk modulus was used. Due to the higher HEL,

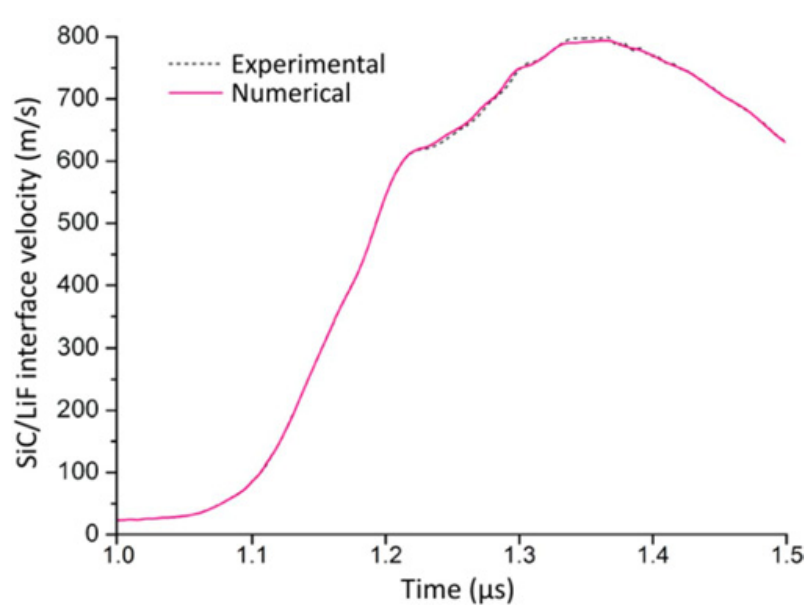

Figure 8. Comparison between experimental and numerical interface velocity signal in the case of the SPS-S grade.

the inelastic part of the loading path obtained with the SPS$\mathrm{S}$ is shorter than the one obtained with the PS-S grade. However, one can anyway see a better work hardening of SPS-S comparing to the PS-S.

Moreover, one can see a very good correlation between the velocity signals obtained experimentally and by simulating the GEPI compression test on SPS-S using the identified plasticity law (cf. Fig. 8).

\section{Conclusions}

In this work, isentropic compression experiments have been performed on two silicon carbide grades using a high pulsed power generator, called GEPI. The use of the GEPI device allows realising a lagrangian analysis with the velocity signals obtained and thus determining the whole loading path in the material. In a first time, the Hugoniot elastic limit (HEL) of the two SiC grades has been determined. The SPS-S grade, having the denser microstructure but composed of big and elongated grains, presents the highest HEL. One can first conclude that the grain size is not the dominating factor on the HEL of silicon carbides and that the densification is probably one more important parameter. In a second time, the lagrangian analysis allows calculating the equivalent stress and identifying a plasticity law. These plasticity laws have then been validated by performing numerical simulations of the compression experiments. It has been observed that the two materials present different work hardening after the elastic limit; work hardening which is more important in the case of the SPS-S grade. However, it is not possible to conclude on the influence of microplasticity and microcracking on the transition between the elastic and the inelastic behaviour and on the work hardening. Shock recovery experiments and microscopic analyses are needed.

This work was supported by DGA (French General Delegation for Armament, French Ministry of Defense). The authors are also grateful to P.-Y. Chanal, J.-J. Royer and P. Rey (CEA GRAMAT) for their technical contribution to this project. 


\section{References}

[1] E.R. Barron, A.L. Alesi, Tech. Report 69-43-CE, U.S. Army Natick Laboratories (1969)

[2] R. Feng, G.R. Raiser, Y.M. Gupta, J. Appl. Phys. 83, 79 (1998)

[3] C.J. Roberson, Ceramic materials and their use in lightweight armour systems, Lightweight armour system symposium (1995)

[4] P.J. Hazell, C.J. Roberson, M. Moutinho, Mat. Design 29, 1497 (2008)
[5] C. Mangeant, F. Lassalle, P. L’Eplattenier, P.L. Héreil, D. Bergues, G. Avrillaud, Shock compression of condensed matter, 1173 (2001)

[6] J. Cagnoux, P. Chartagnac, P.L. Héreil, M. Perez, Ann. Phys. Fr. 12, 451 (1987)

[7] G. Rossiquet, P. Forquin, J.L. Zinszner, F. Barthélémy, Personal Armour System Symposium (2014)

[8] B. Erzar, E. Buzaud, Eur. Phys. J. Special Topics 206, 71 (2012) 